1. Department of Physics,

University of Agriculture, Faisalabad

2. Assistant Professor Cardiology

Faisalabad Institute of Cardiology,

Faisalabad.

3. Department of Physics,

University of Agriculture, Faisalabad

4. Punjab Institute of Nuclear

Medicine

Faisalabad

5. Punjab Institute of Nuclear

Medicine

Faisalabad

6. Punjab Institute of Cardiology

Lahore

Correspondence Address:

Ali Zohaib

Department of Physics,

University of Agriculture, Faisalabad

az_zohaib@yahoo.com

Article received on:

09/01/2016

Accepted for publication:

10/03/2016

Received after proof reading:

$12 / 04 / 2016$

\section{MYOCARDIAL PERFUSION IMAGING (MPI); SUPERIOR CLINICAL PERFORMANCE, A COMPARISON BETWEEN FILTERED BACK- PROJECTION (FBP) AND ASTONISH ITERATIVE RECONSTRUCTION METHOD WITH CARDIAC CATHETERIZATION}

\section{Ali Zohaib', Dr. Muhammad Hamid Saeed ${ }^{2}$, Shaukat Ali Shahid ${ }^{3}$ Muhammad Shahbaz ${ }^{4}$, Faisal Ehsan ${ }^{5}$, Mudassar Iqbal ${ }^{6}$}

\begin{abstract}
Objective: Myocardial perfusion imaging (MPI) with superior quality of images in least acquisition time is imperative for diagnostic purpose. The objective of this study was to compare the Filter Back Projection (FBP) and half time Astonish reconstruction techniques in diagnosis of coronary artery disease using cardiac catheterization as gold standard. Place and period of study: Study was conducted in nuclear cardiology department, at Faisalabad institute of cardiology, Faisalabad from November 2014 to July2015. Material \& Methods: A total of 60patients (56 \pm 19years) underwent gated MPI with Tc-99m Sestamibi and 30 patients of these underwent cardiac catheterization as well. Images were reconstructed with half time Astonish and FBP algorithms. CAD detection, LVEF, perfusion defect comparison using polar maps and diagnostic performance were calculated. Results: Perfusion defects calculated through Astonish and FBP techniques were compared and correlated with coronary angiogram. The sensitivity, specificity and accuracy for detection of CAD in vascular territories (LAD, LCX \& RCA) of the patients were $72 \%, 41 \%$ and $57 \%$ with FBP and $86 \%, 54 \%$ and $71 \%$ with Astonish. The calculated LVEF showed good correlation ( $r=0.8773$ ) between Astonish and cardiac catheterization. Conclusion: There were no significant changes in image quality of Half-time Astonish compared to full-time FBP. Astonish reconstruction showed equal resolution, reduced background noise and radiation dose. Reduced scanning time will improve patient scan quality due to less chance of attenuation.
\end{abstract}

Key words: $\quad$ Single photon emission computed tomography, myocardial perfusion imaging, filtered back projection, left ventricular ejection fraction, coronary artery disease
Article Citation: Zohaib A, Saeed MH, Shahid SA, Shahbaz M, Ehsan F, lqbal M. Spect myocardial perfusion imaging; superior clinical performance, a comparison between filtered back-projection (fbp) and astonish iterative reconstruction method with cardiac catheterization. Professional Med J 2016;23(4):451-459. DOI: 10.17957/TPMJ/16.3245

\section{INTRODUCTION}

Myocardial perfusion SPECT imaging (MPI) with superior quality of images in minimum acquisition time is imperative for diagnostic purpose. Soft tissue attenuation is a noticeable origin of SPECT artifacts $^{1}$, which may result in reduced diagnostic exactness. MPI is a non-invasive procedure utilized for diagnosis of coronary artery disease and so an effective gatekeeper to the cardiac catheterization. $^{2}$ In SPECT, data acquired by multiple projection of the detector around the patient and then raw data reconstructed by using different reconstructed algorithm (FBP, MLEM, OSEM, Astonish etc.) to create a threedimensional (3D) tomogram images. ${ }^{3,4}$
Generally SPECT image quality is based on total acquired counts $^{5}$, configuration of the system and reconstruction algorithms. Noise, artifact and poor spatial resolution in SPECT scan is usually caused by the deficiency of photons detected due to limited scan time, inadequate tracer injected to the patient and soft tissue attenuation. ${ }^{6}$ Image reconstruction by using the appropriate algorithm is important for image quality and diagnostic accuracy. ${ }^{7}$ FBP is still the most commonly used algorithm due to its rapid processing but noise, Compton scatter, soft tissue attenuation and inferior resolution are the main drawback. ${ }^{8}$ FBP contains two stages: first filtering the data and then back projection technique applied on filtered data. ${ }^{9}$ 
An Astonish reconstruction method increases reconstruction accuracy by using double filtering method to reduce noise and enhance uniformity. ${ }^{10}$ Astonish is OSEM (Ordered Subsets Expectation Maximization) iterative technique that reduces statistical noise which can be utilized to reduced injected dose and acquisition time. Astonish algorithm delivers signal-to-noise enhancement, so it generates satisfactory image quality with reduced scan time which on the other hand also reduces motion-induced artifacts. ${ }^{11,12}$ Different kinds of filters used in SPECT image processing generate dissimilar results for example star artifact lessening, noise reduction and signal enhancement. ${ }^{13,14}$

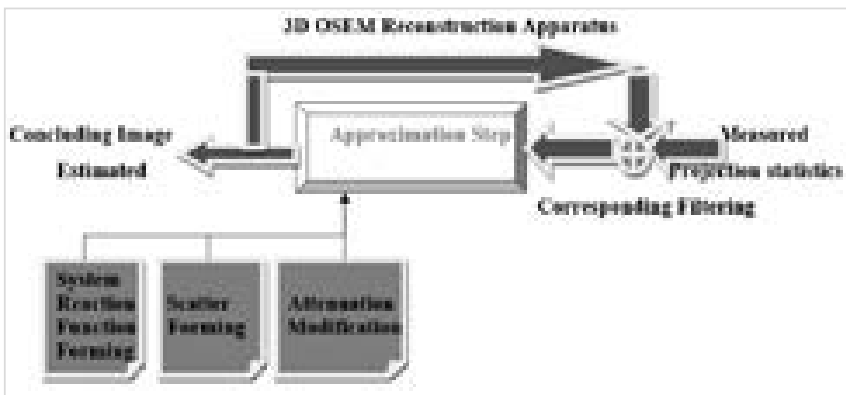

Figure-1. Schematic of Astonish reconstruction using the 3D OSEM iterative method with resolution recovery (system response function modeling) and optional attenuation and scatter modeling.

The Astonish software package uses a threedimensional ordered subsets expectation maximization (3D-OSEM) algorithm for image reconstruction. As inherited from the MLEM algorithm, the 3D-OSEM algorithm models the Poisson noise for the counting statistics during data acquisition, avoiding the long-range noise texture (noise streaks, for example) usually seen in FBP reconstructed images. The three-dimensional implementation allows for the incorporation of 3D resolution recovery during image reconstruction.

\section{MATERIALS AND METHODS}

\section{STUDY POPULATION}

The characteristics of the study population are shown in Table I. We analyzed consecutive 60 patients (39 male and 21 female, $56 \pm 12$ yrs.) who underwent gated MPI with Tc-99m sestamibi. Of theses; 30 patients also underwent cardiac catheterization within two weeks of the study. The patients scan was done from November 2014 through July 2015at Nuclear Cardiology Department, Faisalabad Institute of Cardiology, Faisalabad- Pakistan. All patients underwent two day stress/rest MPI with low-dose. To avoid meddling with the scan investigation, medicine such as beta blockers or calcium channel blockers and caffeine containing compounds were discontinued for at least 24 hours prior to scan.

\begin{tabular}{|c|c|}
\hline Characteristic & Value \\
\hline Age (years). mean $+S D$ & $56+12.2$ \\
\hline Male (\%) & $39(65)$ \\
\hline Female (\%) & $21(35)$ \\
\hline $\mathrm{BMI} \mathrm{kg} / \mathrm{m} 2$. mean $+\mathrm{SD}$ & $25.4+7$ \\
\hline Diabetes (\%) & $59(69)$ \\
\hline Hypertension (\%) & $69(81)$ \\
\hline Hypercholesterolemia (\%) & $62(72)$ \\
\hline Smoker (\%) & $49(57)$ \\
\hline \multicolumn{2}{|l|}{ History of } \\
\hline CAD (\%) & $30(35)$ \\
\hline $\mathrm{Ml}(\%)$ & $39(45)$ \\
\hline CABG (\%) & $09(10)$ \\
\hline
\end{tabular}

\section{RADIOPHARMACEUTICAL AND EXERCISE} STRESS PROTOCOL

All the studies were conducted by using the Tc$99 \mathrm{~m}$ Sestamibi manufactured by PINSTECH. The stress protocol and radiopharmaceutical doses were according to the guidelines of the American Society of Nuclear Cardiology (ASNC).${ }^{16}$ Stress protocol included exercise tolerance test using Bruce and Modified Bruce protocol and in selective cases pharmacologic agents such as Adenosine or Dobutamine were usually used for those patient who were unable to exercise..$^{17,18}$ Radiopharmaceutical doses were on average $327 \mathrm{MBq}(324.4 \pm 17)$ for stress/ rest two days protocol.

DATA ACQUISITION AND RECONSTRUCTION All scans were completed on cardiac dedicated 
CardioMD III (Gamma Camera) using standard FBP and half time Astonish protocol. Low energy high resolution (LEHR) collimation was used and tomography images carried out over $180^{\circ}$ rotation from RAO $45^{\circ}$ to LPO 45 . Total scan time was about 15 minutes in case of FBP and 07 minutes in case of Astonish default.

\section{DATA ANALYSIS}

Myocardial perfusion imaging quality, diagnostic confidence and accuracy for finding of CAD were analyzed by two experienced nuclear physicians and medical physicists. Reconstructed images such as three view and polar map for both stress and rest cardiac tomogram were analyzed visually for poor, equivocal, good and excellent image quality. Sensitivity, specificity and accuracy were calculated for identifying CAD on visual analysis of image reconstructed by the FBP and Astonish algorithms. Moreover LAD, LCx and RCA territories were also analyzed and result compared with cardiac catheterization. Processing software such as Auto-SPECT, 4DM and Quantitative gated SPECT (QGS) were used on gated SPECT data to determine the end-systolic volume (ESV), end-diastolic volume (EDV) and left ventricular ejection fraction (LVEF).

\section{STATISTICAL ANALYSIS}

Data were expressed as mean $\underline{\mathrm{SD}}$ and percentage. System error and degree of agreement were assessed by bland and Altman method. The degree of agreement explained as the mean difference, SD of the difference, limit of agreement and 95\% confidence interval of the mean difference. Reconstructed data by two algorithm were compared using correlation (The value of a correlation coefficient ranges between -1 and 1), regression and paired t-test. Independent data were compared by an independent t-test. A ' $p$ ' value of less than 0.05 was considered statistically significant. Statistically Bland-Altman plots were used to evaluate their degree of agreement. Results were confirmed through cardiac catheterization.

\section{RESULTS}

\section{Clinical result of MPI Interpretations}

Data of 60 consecutive patients ( $56 \pm 12$ yrs., 39 male and 21 female) who underwent gated MPI was reconstructed by the FBP and Astonish algorithms and results analyzed by the two nuclear physicians and medical physicists.

In Figure 2, there was no statistically significant change in interpretive certainty of Half time Astonish compared with FBP. This shows the skill of Astonish to accomplish good image quality of patient's scan with half the counts compared with FBP.
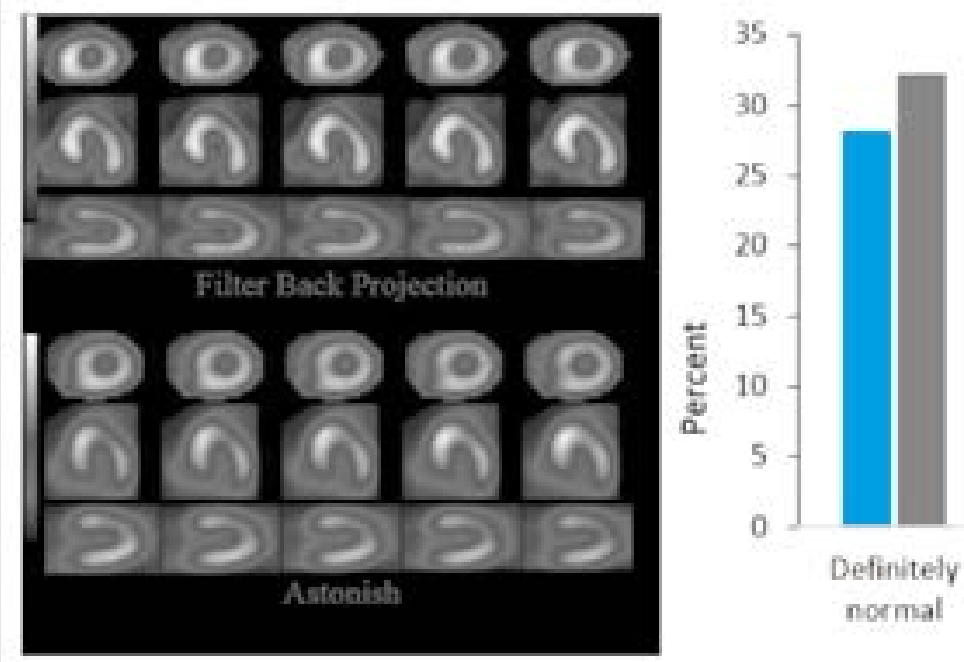

\section{Interpretive Certanity}
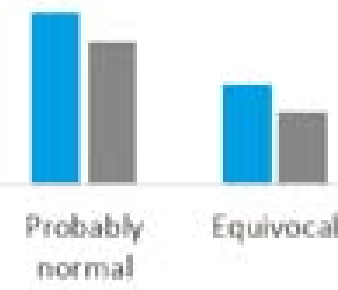

FAP Astanich

Figure-2. Comparison of the interpretative certainty data (short, vertical and horizontal long axis) reconstructed by FBP and Astonish 
Quantitative analysis of myocardial perfusion. For Semi-quantitative visual analysis, the myocardium was separated into three vascular territories: $L A D$, LCx and RCA. The territories area were visually analyzed and correlated with study of cardiac catheterization. The spreading of true positive, true negative, false positive or false negative perfusion scan rendering of the reconstruction algorithm was compared with the cardiac catheterization result (Table II). The sensitivity, specificity and accuracy of vascular territories for the patients were calculated $72 \%, 41 \%$ and $57 \%$ with FBP and $84 \%, 54 \%$ and $71 \%$ with Astonish. The calculated value of LVEF $(r=0.9299)$ showed good correlation between Astonish and cardiac catheterization.


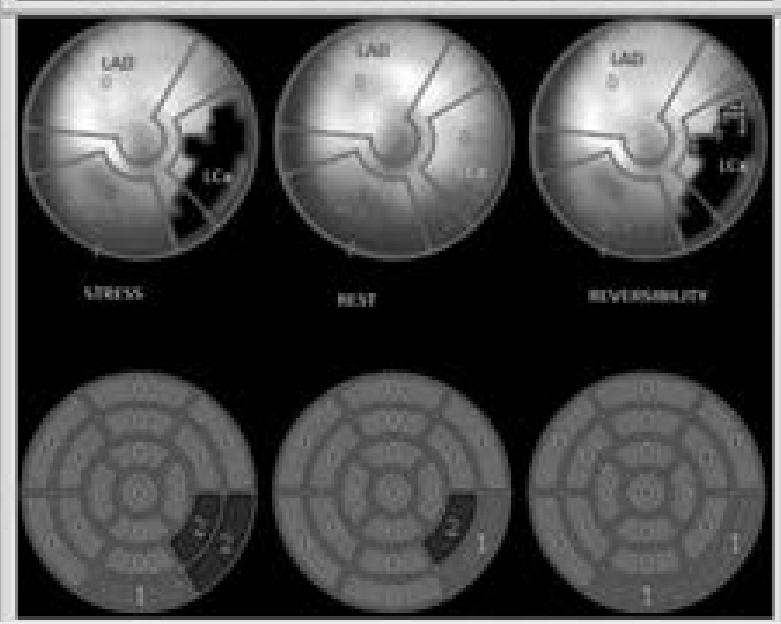

(A)

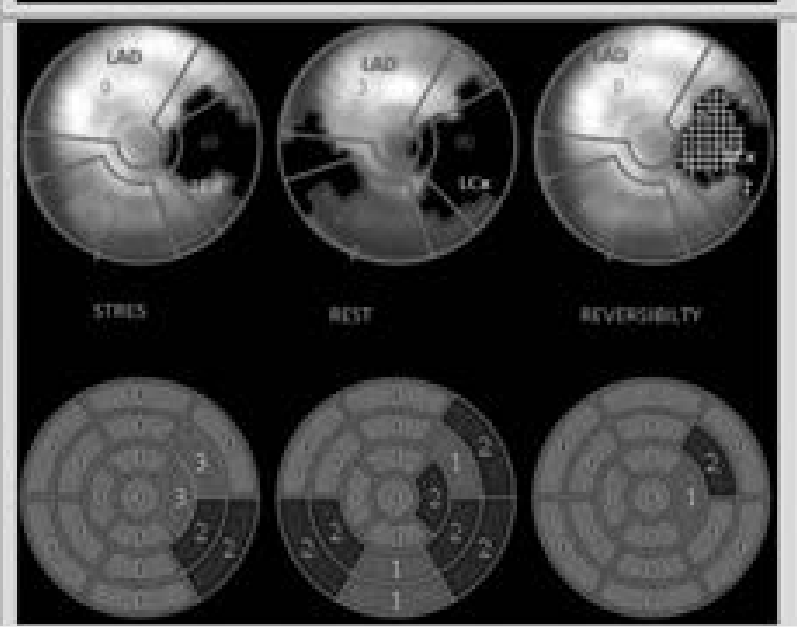

(B)

Figure-3. Stress/Rest Perfusion defect (short, vertical and horizontal long axis), Cardiac vessel territories and summed score calculated by (A) FBP and (B) Half time Astonish reconstruction algorithm. 
A 60 years old male patient with history of chest pain and three vessel coronary artery disease underwent MPI for the assessment of reversible myocardial ischemia. Both the physicians conclude that the data reconstructed by the FBP showed mild reversible myocardial ischemia involving the lateral and inferolateral walls. No evidence of reversible ischemia in LAD and RCA territories. Slight reversibility in LCx (52\% normal, 39\% fixed, Reverse 09\%) and EF: $49 \%$. The data reconstructed by the half time astonish algorithm showed that reduced tracer uptake in the septal wall and mild reversible myocardial ischemia involving the lateral and inferolateral walls. No evidence of reversible ischemia in LAD and RCA territories. Mild reversibility in LCx (31\% normal, $21 \%$ fixed, Reverse $48 \%$ ) and EF: $54 \%$. The cardiac catheterization showed that LAD: $60 \%$ mid stenosis. RCA: Dominate vessel, total mid Occlusion LCx: mid disease involving/Total proximal occlusion EF: $60 \%$. Figure 3(B) image was reconstructed with Astonish having no loss in diagnostic confidence level as compared to cardiac catheterization which is the gold standard. Figure $3(A)$ image was reconstructed by FBP that shows adequate diagnostic accuracy as compared to Astonish.

\section{CHARACTERIZATION OF MYOCARDIAL PERFUSION DEFECTS}

The resultant polar maps of myocardial perfusion were allocated to one of the three major territories by standard pattern. Perfusion defects were automatically computed for the normal, fixed and severity with FBP and Astonish SPECT reconstruction algorithms (Figure 3). Tracer distribution and perfusion in the cardiac territory is shown in Table III.

\begin{tabular}{|l|c|}
\hline Characteristic (\%) & All Patients $\mathrm{n}=30$ \\
\hline Single vessel CAD & $40.1 \%$ \\
\hline Double vessel CAD & $28.1 \%$ \\
\hline Triple vessel CAD & $15.3 \%$ \\
\hline Significant disease in LAD territory & $19.7 \%$ \\
\hline Significant disease in RCA territory & $8.4 \%$ \\
\hline Significant disease in LCx territory & $11.6 \%$ \\
\hline Total occlusion in LAD territory & $2.1 \%$ \\
\hline Total occlusion in RCA territory & $1.3 \%$ \\
\hline Total occlusion in LCx territory & $2.2 \%$ \\
\hline \multicolumn{2}{|c|}{ Table-II. Number of diseased vesselsin cardiac } \\
\hline \multicolumn{2}{|c|}{ catheterization results } \\
\hline
\end{tabular}

\begin{tabular}{|l|c|c|c|c|c|c|}
\hline \multicolumn{1}{|c|}{ Method } & Territory & Normal & Mild & Moderate & Severe & Fixed \\
\hline \multirow{3}{*}{ FBP } & LAD & 25 & 10 & 09 & 10 & 06 \\
\hline \multirow{3}{*}{ Astonish } & RCA & 39 & 11 & 07 & 02 & 01 \\
\hline \multirow{3}{*}{} & LCx & 26 & 13 & 11 & 08 & 02 \\
& LAD & 21 & 11 & 09 & 12 & 07 \\
\hline & RCA & 31 & 08 & 11 & 06 & 04 \\
\hline
\end{tabular}

Table-III. Myocardial perfusion defect in territories LAD, LCX and RCA territories reconstructed by FBP and Astonish algorithm by using 4DM software

\begin{tabular}{|l|c|c|c|c|c|c|}
\hline \multicolumn{1}{|c|}{ Method } & Territory & Normal & Mild & Moderate & Severe & Fixed \\
\hline \multirow{3}{*}{ FBP } & LAD & 25 & 10 & 09 & 10 & 06 \\
\hline \multirow{3}{*}{ Astonish } & RCA & 39 & 11 & 07 & 02 & 01 \\
\hline \multirow{3}{*}{} & LCX & 26 & 13 & 11 & 08 & 02 \\
\hline & LAD & 21 & 11 & 09 & 12 & 07 \\
\hline & RCA & 31 & 08 & 11 & 06 & 04 \\
\hline
\end{tabular}

Table-III. Myocardial perfusion defect in territories LAD, LCX and RCA territories reconstructed by FBP and Astonish algorithm by using 4DM software 


\begin{tabular}{|c|c|c|c|c|c|c|}
\hline & \multicolumn{6}{|c|}{ Number of Patients (\%) } \\
\hline & \multicolumn{3}{|c|}{ FBP } & \multicolumn{3}{|c|}{ Astonish } \\
\hline & LAD & LCx & RCA & LAD & LCx & RCA \\
\hline TP & $15(50)$ & $10(33)$ & $8(27)$ & $18(60)$ & $12(40)$ & $13(43)$ \\
\hline FP & $07(23)$ & $12(40)$ & $07(23)$ & $04(14)$ & $09(30)$ & $07(23)$ \\
\hline TN & $05(17)$ & $06(20)$ & $07(23)$ & $07(23)$ & $07(23)$ & $07(23)$ \\
\hline FN & $03(10)$ & $02(07)$ & $08(27)$ & $01(03)$ & $02(07)$ & $03(10)$ \\
\hline
\end{tabular}

Table-V. MPI interpretation using FBP and Astonish against cardiac catheterization considering as gold standard. $F N=$ false negative; $F P=$ false positive; $T N=$ true negative; $T P=$ true positive.

\begin{tabular}{|c|c|c|c|}
\hline Disease prevalence & LAD & LCx & RCA \\
\hline FBP & $60 \%$ & $40 \%$ & $53 \%$ \\
\hline AS & $64 \%$ & $46 \%$ & $54 \%$ \\
\hline
\end{tabular}

Table-VI. Disease prevalence of myocardium vascular territories calculated by FBP, AS and ASM

\section{ASSESSMENT BETWEEN MPI AND CARDIAC} CATHETERIZATION

All myocardial perfusion scan results (normal, mild, moderate severe and fixed) in table III were compared with cardiac catheterization result in Table II. The true positive (TP), false positive (FP), true negative (TN), or false negative (FN) perfusion defects distribution rendering to the reconstruction method and stenosis localization compared with the cardiac catheterization is shown in Table IV.

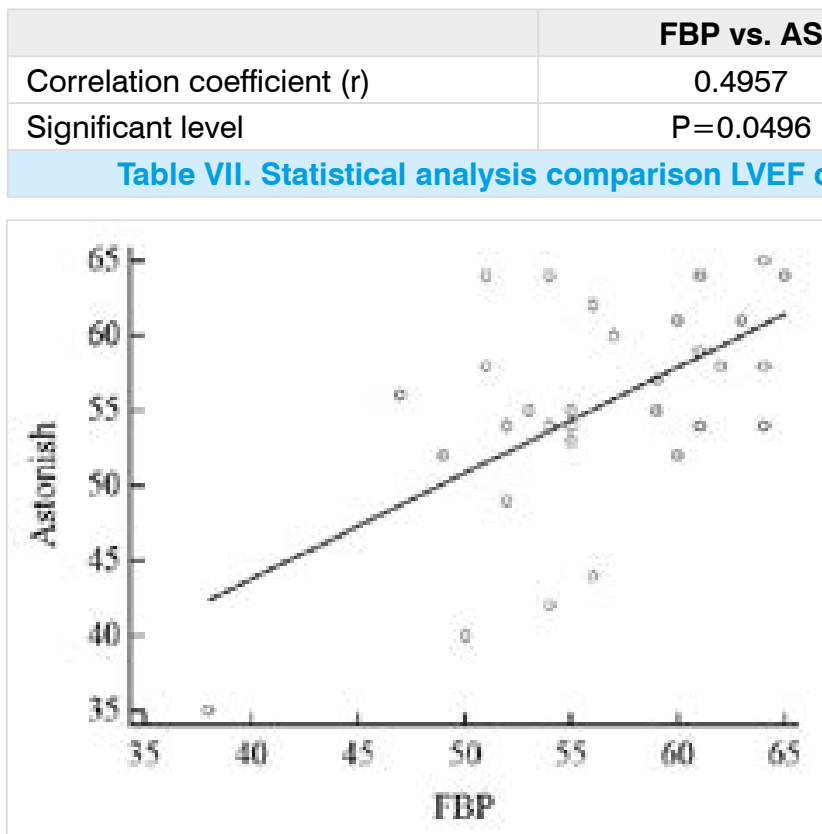

In Figure 4, scatter plot showed that there is strongly poor correlation $(r=0.4957)$ of LVEF calculated by FB and Astonish.
Figure 4. Correlation of LVEF between FB and Astonish

Professional Med J 2016;23(4): 451-459.

\section{DIAGNOSTIC ACCURACY}

There was no statistical change in the diagnostic accuracy of Half time data reconstructed with Astonish and full time with filtered back projection.

\section{FUNCTIONAL RESULTS}

Scatter plot and Bland-Altman plot for Ejection Fraction between gated MPI of Astonish and FBP algorithm were compared with cardiac catheterization result. The results of Bland-Altman are summarized in Table VII.

\begin{tabular}{|l|c|c|c|}
\hline Correlation coefficient $(r)$ & 0.4957 & 0.5427 & 0.8773 \\
\hline Significant level & $\mathrm{P}=0.0496$ & $\mathrm{P}=0.801$ & $\mathrm{P}=0.993$ \\
\hline
\end{tabular}

FBP vs. CC

$\mathrm{P}=0.801$
CC vs. AS

$\mathrm{P}=0.993$

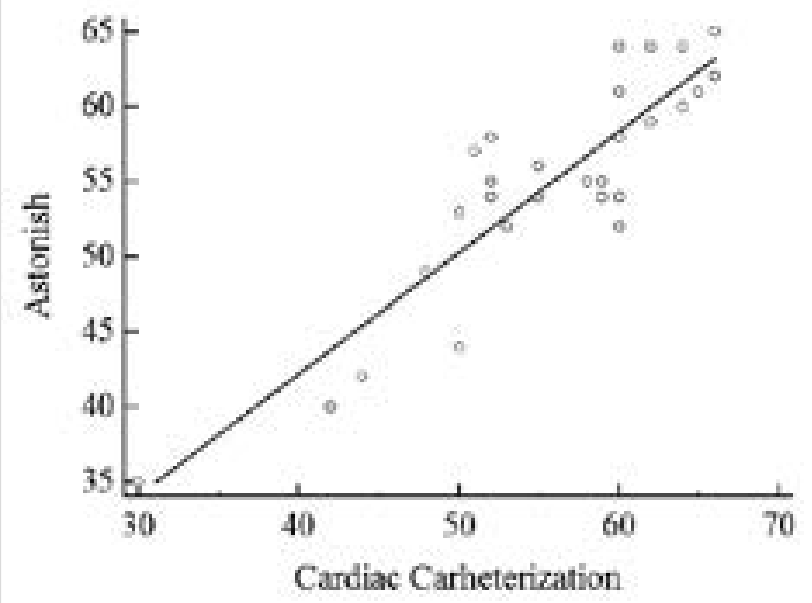

Figure 5. Correlation of LVEF between Cardiac Catheterization and Astonish

In Figure 5, scatter plot showed that there is good correlation ( $r=0.8773$ ) of LVEF calculated by Cardiac Catheterization and Astonish. 


\section{LVEF Comarision}

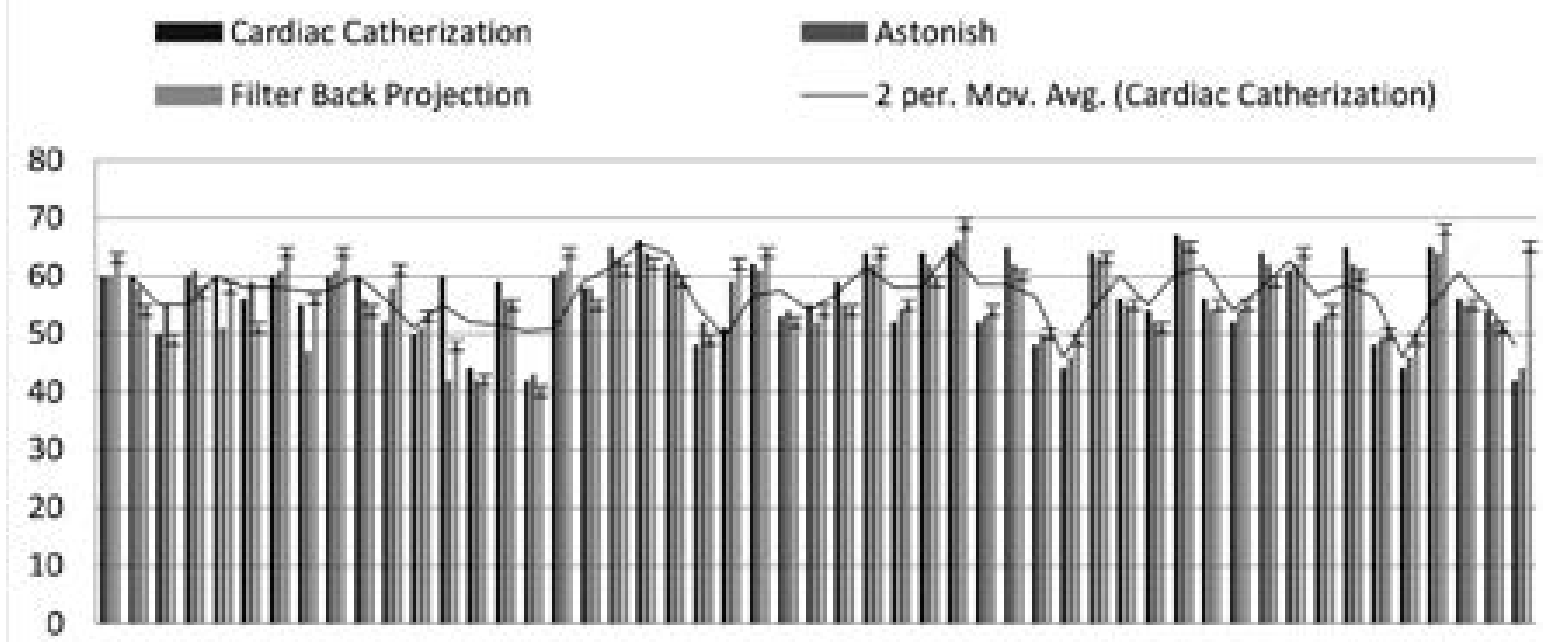

Figure-7. LVEF comparison among Astonish, FBP and cardiac catheterization

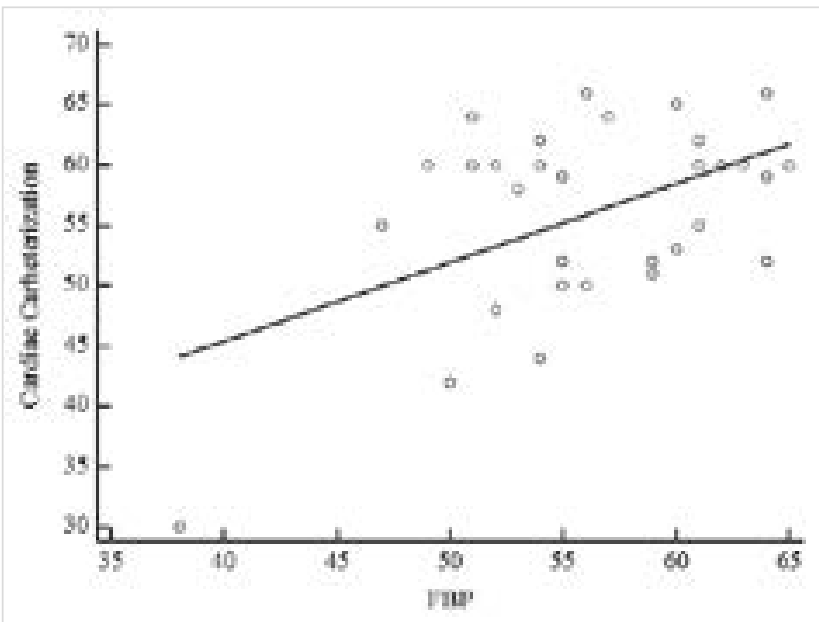

Figure-6. Correlation of LVEF between FBP and Cardiac Catheterization

In Figure 6, scatter plot showed that there is poor correlation $(r=0.5427)$ LVEF calculated by Cardiac Catheterization and FBP.

Figure 7 shows that LVEF values calculated by cardiac catheterization and Astonish are more precise rather than FBP.

\section{DISCUSSION}

Minimizing the acquisition time may possibly increase patient throughput, increase camera efficiency and decrease costs. Moreover, time reducing also produces image noise. ${ }^{21}$ Half time image reconstruction algorithm can generate reconstructed images with enhanced spatial resolution and reduced noise level developed newly available. ${ }^{22}$ In previous analysis of gated SPECT MPI, data were reconstructed by FBP and newly iterative produced basically showed similar results. ${ }^{23}$ Astonish is technologically advanced SPECT image reconstruction algorithm that contains correction to enhance image quality with half scan time. Astonish based on the OSEM reconstruction algorithm using built-in noise decrease methods throughout the iterative procedure. In integrating corrections for photon scatter offers a more detailed image of the counts from lesions that are at different depths inside the patient. To minimize the statistical noise throughout the reconstruction procedure, Astonish uses exclusive noise lessening technique for smoothing both the assessed projection data and the measured projection data internally throughout the data reconstruction by an optional Henning filter. The significant aspects that disturbs the quality of medical SPECT images is choosing appropriate filter. Filtering of image is a smoothness procedure for noise elimination and resolution recovery. Furthermore, cut-off frequency is also significant so as to decrease noise. No filter till now is perfect and there is no exact single filter for entirely applications. 
In this study we used FBP and Astonish algorithms. There was no statistical change in diagnostic accuracy. Astonish reconstruction algorithms produced detailed images of radioactive distribution and look to be more sensitive than FBP algorithm. By using the astonish algorithm with half time and low dose allow the patient to feel comfort during scan and is less affected by the radiation dose as compared to FBP by which patients felt discomfort during scan resulting in artifacts, attenuation effect and poor resolution. Low radiation dose implements the ALRA principle so minimum chance to damage the DNA, because ionization radiation damages the DNA. Low dose will also affect cost and less use of radioactive material as the availability of radioactive material is rare. So short scan time is probable to decrease the rate of patient motion and less of motion artifacts. This study showed that the Astonish gives higher accuracy in diagnosis than FBP for detecting CAD in LAD, RCA and LCx territory.

Moreover, Astonish with low dose $(300 \pm 21) \mathrm{MBq}$ or half time was unable to yield the best image quality for the overweight patients $(118 \pm 23)$ due to breast attenuation, lateral wall thickness of obese patients, and other attenuation factor. Artifacts and attenuation due to hepatobiliary effect were more significance in case of astonish rather than in FBP which may be due to half scan time or increased number of iteration in Astonish. So raw data were reviewed and checked the extra cardiac activity, as the extra cardiac activity generate poor image quality.

Furthermore, in former scan results, patient felt discomfort due to long scan time and because of attenuation. Consequently to eliminate the doubt of attenuation we have to perform the rest scan of the patient and again patient suffers from increase radiation dose as well as more time consuming. So FBP increases the chance of rest and confuse the physicians for exact reporting due to attenuation effect.

\section{CONCLUSIONS}

The images showed that the practice of half time Astonish algorithm enhance image quality and may possibly be useful to minimize the scan acquisition time without compromising in diagnostic accuracy. Proper parameters selection supports the physician in result interpretation; precisediagnosis thus minimizes the need of rest scan and less chance of attenuation.

Copyright $(10$ March, 2016.

\section{REFERENCES}

1. Doukky R, Rahaby M, and Amin A.P, "Soft Tissue Attenuation Patterns Associated with Supine Acquisition SPECT Myocardial Perfusion Imaging: A Descriptive Study," The Open Cardiovascular Medicine Journal, 2010; 6: 33-37.

2. Hachamovitch R, andCarli M.D, "Nuclear cardiology will remain the "gatekeeper" over CT angiography, "Journal, 2007;14: 634-644.

3. Gaemperli O, Schepis $T$ and Kalff et V. "Validation of a new cardiac image fusion software for threedimensional integration of myocardial perfusion SPECT and stand-alone 64-slice CT angiography, "European Journal of Nuclear Medicine and Molecular Imaging, 2007;7:1097-1106.

4. Heller G.V,Bateman T.M, Cullom S.J et al.,"“Improved clinical performance of myocardial perfusion SPECT imaging using Astonish iterative reconstruction, "Philips.

5. He B and Frey B.E,"Effects of shortened acquisition time on accuracy and precision of quantitative estimates of organ activity,"Medical Physics 2010; 37: 1807-15.

6. Saad I.E, "Evaluation of the Image Contrast with a New Depth-Dependent Collimator Resolution Iterative Reconstruction Method," Egyptian Journal of Nuclear Medicine, 2010;2: 55-62.

7. Hoorn R.R,Vriens D, Postema W, et al., "The influence of SPECT reconstruction algorithms on image quality and diagnostic accuracy in phantom measurements and 99mTc-sestamibi parathyroid scintigraphy," Nuclear Medicine Communication,2014; 35:64-72.

8. Katua A.M, Ankrah A.O, Vorster M, "Optimization of Ordered Subset Expectation Maximization Reconstruction for Reducing Urinary Bladder Artifacts in Single-photon Emission Computed Tomography Imaging,"World Journal Nuclear Medicine.vol. 2011;10: 3-8.

9. Cherry S.R, Sorenson J.A, and Phelps M.E, "Physics in Nuclear Medicine, Saunders," Philadelphia, Pa, USA, 
2003.

10. Depuey E.G, "Advances in Cardiac Processing Software," Seminars in Nuclear Medicine,2014;4:252-273.

11. Seret A, Nguyen D, and Bernard C, "Quantitative capabilities of four state-of-the-art SPECT-CT cameras," EJNMMI Research 2012: 2: 1-19.

12. Travin M.I, "Cardiac Camera," Seminars in Nuclear Medicine, 2011; 3:182-201.

13. Elhendy A, Schinkel A.F, Domburg R.T et al.,"Prognostic Stratification of Obese Patients by Stress $99 \mathrm{~m}$ - TcTetrofosmin Myocardial Perfusion Imaging," Journal of Nuclear Medicine,2006; 47:1302-1306.

14. Laere K.V, Koole M, Lemahieu I, and Dierckx R, "Image filtering in single-photon emission computed tomography: Principles and applications," Computerized Medical Imaging and Graphics, 2011;2: 127-133.

15. Baggish A.L,Boucher C.A, "Contemporary Reviews in Cardiovascular Medicine Radiopharmaceutical Agents for Myocardial Perfusion Imaging,"Circulation, 2008; 118:1668-1674.

16. Hill $\mathrm{J}$ and Timmis A, "Exercise tolerance testing,"BMJ, 2002;324: 1084-1087.

17. Henzlova M.J, Manuel D. Cerqueira, J. J. Mahmarian
andS. Yao,"Stress protocols and tracers," Journal of Nuclear Cardiology,2006:13: 80-90.

18. Fathala A, "Myocardial Perfusion Scintigraphy: Techniques, Interpretation, Indications and Reporting,"Annals of Saudi Medicine. 2011; 6: 625634.

19. Abidov A, Bax J.J, Hayes s.W et al.,"Integration of Automatically Measured Transient Ischemic Dilation Ratio into Interpretation of Adenosine Stress Myocardial Perfusion SPECT for Detection of Severe and Extensive CAD,"Journal of Nuclear Medicine, 2004; 45:1999-2007.

20. Elhendy A, Bax J, Poldermans D, "Dobutamine Stress Myocardial Perfusion Imaging in Coronary Artery Disease,"Journal of Nuclear Medicine2002:1: 16341646.

21. Ali L, Ruddy T.D, Almgrahi A,"Half-time SPECT myocardial perfusion imaging with attenuation correction,".Journal Nuclear Medicine 2009; 50:,55462.

22. P. Knoll, D.Kotalova, G.Kochle et al.,"Comparison of advanced iterative reconstruction methods for SPECT/CT.Z," Medical Physics, 2012;22: 58-69.

23. Li D, Zhou Y, Feng.J , Yuan Det al., "Impact of image reconstruction on phase analysis of ECG-gated myocardial perfusion SPECT studies,"Nuclear Medicine Communication,2010;30: 700-705

\section{AUTHORSHIP AND CONTRIBUTION DECLARATION}

\begin{tabular}{|c|l|l|l|}
\hline Sr. \# & \multicolumn{1}{|c|}{ Author-s Full Name } & \multicolumn{1}{|c|}{ Contribution to the paper } & Author=s Signature \\
\hline 1 & Ali Zohaib & $35 \%$ & \\
2 & Dr. M. Hamid Saeed & $20 \%$ \\
3 & Shaukat Ali Shahid & $15 \%$ & \\
4 & Muhammad Shahbaz & $10 \%$ & \\
5 & Faisal Ehsan & $10 \%$ & \\
6 & Mudassar lqbal & $10 \%$ & \\
\hline
\end{tabular}

